\title{
A generalization of Reilly's formula and its applications to a new Heintze-Karcher type inequality
}

\author{
Guohuan Qiu * $^{*} \quad$ Chao Xia ${ }^{\dagger}$
}

\begin{abstract}
In this paper, we prove a generalization of Reilly's formula in 10. We apply such general Reilly's formula to give alternative proofs of the Alexandrov's Theorem and the Heintze-Karcher inequality in the hemisphere and in the hyperbolic space. Moreover, we use the general Reilly's formula to prove a new Heintze-Karcher inequality for Riemannian manifolds with boundary and sectional curvature bounded below.
\end{abstract}

Keywords: Reilly's formula, constant mean curvature, Rigidity, HeintzeKarcher inequality.

\section{Introduction}

In a celebrated paper [10, Reilly proved an integral formula for compact Riemannian manifolds with smooth boundary. To be precise, let us first give our notations. Throughout this paper, Let $\left(\Omega^{n}, g\right)$ be an $n$-dimensional compact Riemannian manifold with smooth boundary $M$. We denote by $\bar{\nabla}, \bar{\Delta}$ and $\bar{\nabla}^{2}$ the gradient, the Laplacian and the Hessian on $\Omega$ respectively, while by $\nabla$ and $\Delta$ the gradient and the Laplacian on $M$ respectively. Let $\nu$ be the unit outward normal of $M$. We denote by $h(X, Y)=g\left(\bar{\nabla}_{X} \nu, Y\right)$ and $H=\frac{1}{n-1} \operatorname{tr}_{g} h$ the second fundamental form and the (normalized) mean curvature (with respect to $\nu$ ) of $M$ respectively. Let $d \Omega$ and $d A$ be the canonical measure of $\Omega$ and $M$ respectively. Let Sect and Ric be the sectional curvature and the Ricci curvature tensor of $\Omega$ respectively.

Given a smooth function $f$ on $\Omega$, we denote $z=\left.f\right|_{M}$ and $u=\bar{\nabla}_{\nu} f$. Reilly's formula [10] states that

$$
\begin{aligned}
& \int_{\Omega}\left\{(\bar{\Delta} f)^{2}-\left|\bar{\nabla}^{2} f\right|^{2}-\operatorname{Ric}(\bar{\nabla} f, \bar{\nabla} f)\right\} d \Omega \\
& =\int_{M}\left\{2 u \Delta z+(n-1) H u^{2}+h(\nabla z, \nabla z)\right\} d A .
\end{aligned}
$$

Reilly's formula (11) has numerous applications. For example, in [10] Reilly himself applied it to prove a Lichnerowicz type sharp lower bound for the first

\footnotetext{
${ }^{*}$ Department of Mathematics, University of Science and Technology of China, Hefei, P. R. China Email:guohuan@mail.ustc.edu.cn.

${ }^{\dagger}$ Max-Planck-Institut für Mathematik in den Naturwissenschaften, Inselstr. 22, D-04103, Leipzig, Germany Email: chao.xia@mis.mpg.de.
} 
eigenvalue of the Laplacian on manifolds with boundary and reprove Alexandrov's rigidity theorem for embedded hypersurfaces with constant mean curvature in $\mathbb{R}^{n}$. Other applications can be found for instance in [5, 9, 12].

In [12] Ros used Reilly's formula to prove the following integral inequality, which was applied to show Alexandrov's rigidity theorem for high order mean curvatures.

Theorem A. (Ros [12]) Let $\left(\Omega^{n}, g\right)$ be a compact $n$-dimensional Riemannian manifold with smooth boundary $M$ and non-negative Ricci curvature. Let $H$ be the mean curvature of $M$. If $H$ is positive everywhere, then

$$
\int_{M} \frac{1}{H} d A \geq n \operatorname{Vol}(\Omega) .
$$

The equality in (2) holds if and only if $\Omega$ is isometric to an Euclidean ball.

For $\Omega \subset \mathbb{R}^{n}$, inequality (2) is essentially contained in the paper of Heintze and Karcher [7. Ros' proof of Theorem A based on the Reilly's formula (1) and a suitable Dirichlet boundary value problem. Later, Montiel and Ros 8 gave an alternative proof of Theorem $\mathrm{A}$ in the case $\Omega \subset \mathbb{R}^{n}$ based on the ideas of Heintze and Karcher [7, so that they had an alternative proof of Alexandrov's theorem in $\mathbb{R}^{n}$. Using the same idea of [7, they also showed Alexandrov type theorem for constant higher order mean curvature embedded hypersurfaces in the hemi-sphere $\mathbb{S}_{+}^{n}$ or the hyperbolic space $\mathbb{H}^{n}$. However, they could not prove a similar inequality as (2) in $\mathbb{S}_{+}^{n}$ or $\mathbb{H}^{n}$.

Quite recently, in order to study the Alexandrov type rigidity problem in general relativity, Brendle [2] gave a version of Theorem A in $\mathbb{S}_{+}^{n}$ and $\mathbb{H}^{n}$, more generally, in a large class of warped product spaces, including the Schwarzschild manifold.

For $\mathbb{S}_{+}^{n}$ and $\mathbb{H}^{n}$, Brendle's result states as follows.

Theorem B. (Brendle 2]) Let $\Omega^{n} \subset \mathbb{H}^{n}$ ( $\mathbb{S}_{+}^{n}$ resp.) be a compact $n$-dimensional domain with smooth boundary $M$. Let $H$ be the normalized mean curvature of $M$. Let $V(x)=\cosh _{\operatorname{dist}_{\mathbb{H}^{n}}}(x, 0)\left(\cos \operatorname{dist}_{\mathbb{S}^{n}}(x, 0)\right.$ resp. $)$. If $H$ is positive everywhere, then

$$
\int_{M} \frac{V}{H} d A \geq n \int_{\Omega} V d \Omega .
$$

The equality in (3) holds if and only if $\Omega$ is isometric to a geodesic ball.

As mentioned before, Brendle proved (3) for more general warped product spaces. Recently, his inequality has many interesting applications in general relativity, see for instance [3, 4, 6.

Brendle's method is quite different from Ros'. He used a geometric flow, along which the quantity $\int_{M} \frac{V}{H} d A$ is monotone non-increasing, to prove Theorem B. It is a natural problem to ask whether there is a Reilly-Ros type proof for Theorem B. This is the motivation of this paper.

In this paper, we first prove the following general Reilly's formula.

Theorem 1.1. Let $V: \bar{\Omega} \rightarrow \mathbb{R}$ be a given a.e. twice differentiable function. Given a smooth function $f$ on $\Omega$, we denote $z=\left.f\right|_{M}$ and $u=\bar{\nabla}_{\nu} f$. Let $K \in \mathbb{R}$. 
Then we have the following identity:

$$
\begin{aligned}
& \int_{\Omega} V\left((\bar{\Delta} f+K n f)^{2}-\left|\bar{\nabla}^{2} f+K f g\right|^{2}\right) d \Omega \\
= & \int_{M} V\left(2 u \Delta z+(n-1) H u^{2}+h(\nabla z, \nabla z)+(2 n-2) K u z\right) d A \\
& +\int_{M} \bar{\nabla}_{\nu} V\left(|\nabla z|^{2}-(n-1) K z^{2}\right) d A \\
& +\int_{\Omega}\left(\bar{\nabla}^{2} V-\bar{\Delta} V g-(2 n-2) K V g+V R i c\right)(\bar{\nabla} f, \bar{\nabla} f) d \Omega \\
& +(n-1) \int_{\Omega}\left(K \bar{\Delta} V+n K^{2} V\right) f^{2} d \Omega .
\end{aligned}
$$

When $V \equiv 1$ and $K=0$, (4) reduces to Reilly's formula (11). We are interested in some other choices of $V$ in this paper, particularly, $V(x)=\cosh r(x)$ or $\cos r(x)$, where $r(x)=\operatorname{dist}_{g}(x, p)$ for some fixed point $p$ in $\Omega$.

Similar as Reilly [10, (44) can be applied to reprove Alexandrov's theorem in $\mathbb{S}_{+}^{n}$ and $\mathbb{H}^{n}$, which is due to Alexandrov [1.

Theorem 1.2 (Alexandrov, 1]). Let $M$ be an embedded closed hypersurface in $\mathbb{S}_{+}^{n}$ or $\mathbb{H}^{n}$ with constant mean curvature $H$. Then $M$ must be a geodesic sphere.

Based on (4), we are also able to give an alternative proof of Theorem B. Moreover, our approach enables us to give a new Heintze-Karcher inequality for more general Riemannian manifolds with boundary.

Theorem 1.3. Let $\left(\Omega^{n}, g\right)$ be a n-dimensional compact Riemannian manifold with smooth boundary $M$. Assume that the sectional curvature of $\Omega$ has a lower bound Sect $\geq-1$. Let $H$ be the normalized mean curvature of $M$. Let $V(x)=\cosh r(x)$, where $r(x)=\operatorname{dist}_{g}(x, p)$ for some fixed point $p$ in $\Omega$. If $H$ is positive everywhere, then

$$
\int_{M} \frac{V}{H} d A \geq \int_{M} \bar{\nabla}_{\nu} V d A=\int_{\Omega} \bar{\Delta} V d \Omega .
$$

The equality in (5) holds if and only if $\Omega$ is a geodesic ball in a space form whose sectional curvature is -1 .

Note that if $\Omega$ is of constant sectional curvature -1 , then $\bar{\Delta} V=n V$. Then Theorem 1.3 reduces to Theorem $\mathrm{B}$ for the case $\Omega \subset \mathbb{H}^{n}$. We remark that the proof of Theorem 1.3 also applies to the case $\Omega \subset \mathbb{S}_{+}^{n}$ to show (3). Therefore, we give an alternative proof of Theorem B.

Inequality (5) is motivated by Brendle's inequality for warped product spaces 2. However, except for the case of constant curvature manifolds, they are not the same. First, in Theorem 1.3, we assume a lower bound of sectional curvature for Riemannian manifolds. Brendle [2] assumed the warped product structure for Riemannian manifolds and some conditions on the warped product function. Second, the equality case in our inequality can only occur for constant curvature manifolds. The equality case in Brendle's inequality can occur for the warped product spaces he considered.

Comparing Theorem 1.3 with Theorem A, we want to ask whether Theorem 1.3 holds if Ric $\geq-(n-1) g$ ? Also if the right hand side of (5) is replaced by $n \int_{\Omega} V d \Omega$, is (5) still true? Note that for Ric $\geq-(n-1) g$, we have $\bar{\Delta} V \leq n V$. Hence the inequality $\int_{M} \frac{V}{H} d A \geq n \int_{\Omega} V d \Omega$ is stronger than (5). 
Our method is based on the general Reilly's formula (44) and the following Dirichlet boundary value problem

$$
\left\{\begin{array}{rlll}
\bar{\Delta} f & = & n f & \text { in } \Omega \\
f & = & c & \text { on } M
\end{array}\right.
$$

for some real constant $c>0$. The existence and the regularity of solutions to (6) follows from standard theory of second order elliptic PDEs.

The paper is organized as follows. Section 2 is devoted to prove the general Reilly's formula (44). In Section 3, we use (4) to give an alternative proof of Theorem 1.2. In Section 4, we use (4) to prove the new Heintze-Karcher inequality, Theorem 1.3

\section{General Reilly's formula}

For simplicity, We use $f_{i}, f_{i j}, \cdots$ and $f_{\nu}$ to denote covariant derivatives and normal derivative of a function $f$ with respect to $g$ respectively.

By integration by parts and Ricci identity, we have

$$
\begin{aligned}
& \int_{\Omega} V\left|\bar{\nabla}^{2} f\right|^{2} d \Omega=\int_{\Omega} V \sum_{i, j=1}^{n} f_{i j} f_{i j} d \Omega \\
= & \int_{M} V \sum_{i=1}^{n} f_{i \nu} f_{i} d A-\int_{\Omega} \sum_{i, j=1}^{n} V_{j} f_{i j} f_{i} d \Omega-\int_{\Omega} V \sum_{i, j=1}^{n} f_{i j j} f_{i} d \Omega \\
= & \int_{M} V \sum_{i=1}^{n} f_{i \nu} f_{i} d A-\int_{\Omega} \sum_{j=1}^{n} V_{j}\left(\frac{1}{2}|\bar{\nabla} f|^{2}\right)_{j} d \Omega \\
& -\int_{\Omega} \sum_{i=1}^{n} V\left((\bar{\Delta} f)_{i}+\sum_{j=1}^{n} R_{i j} f_{j}\right) f_{i} d \Omega \\
= & \int_{M} V \sum_{i=1}^{n} f_{i \nu} f_{i} d A-\int_{M} \frac{1}{2}|\bar{\nabla} f|^{2} V_{\nu} d A+\int_{\Omega} \frac{1}{2}|\bar{\nabla} f|^{2} \bar{\Delta} V d \Omega \\
& -\int_{M} V \bar{\Delta} f f_{\nu} d A+\int_{\Omega} V(\bar{\Delta} f)^{2} d \Omega+\int_{\Omega} \bar{\Delta} f \sum_{i=1}^{n} V_{i} f_{i} d \Omega \\
& -\int_{\Omega} V \sum_{i, j=1}^{n} R_{i j} f_{i} f_{j} d \Omega .
\end{aligned}
$$

We also have

$$
\int_{\Omega} V f \bar{\Delta} f d \Omega=\int_{M} V f f_{\nu} d A-\int_{\Omega}\left(V|\bar{\nabla} f|^{2}+\sum_{i=1}^{n} V_{i} f_{i} f\right) d \Omega .
$$


Using (77) and (8), we obtain

$$
\begin{aligned}
& \int_{\Omega} V\left((\bar{\Delta} f+K n f)^{2}-\left|\bar{\nabla}^{2} f+K f g\right|^{2}\right) d \Omega \\
= & \int_{\Omega} V\left((\bar{\Delta} f)^{2}-\left|\bar{\nabla}^{2} f\right|^{2}\right) d \Omega \\
& +(2 n-2) K \int_{\Omega} V f \bar{\Delta} f d \Omega+n(n-1) K^{2} \int_{\Omega} V f^{2} d \Omega \\
= & \int_{M} V \bar{\Delta} f f_{\nu}+\frac{1}{2}|\bar{\nabla} f|^{2} V_{\nu}-V \sum_{i=1}^{n} f_{i \nu} f_{i}+(2 n-2) K V f f_{\nu} d A \\
& +\int_{\Omega}-\frac{1}{2}|\bar{\nabla} f|^{2} \bar{\Delta} V-\bar{\Delta} f \sum_{i=1}^{n} V_{i} f_{i}+V \sum_{i, j=1}^{n} R_{i j} f_{i} f_{j} d \Omega \\
& -(2 n-2) K \int_{\Omega}\left(V|\bar{\nabla} f|^{2}+\sum_{i=1}^{n} V_{i} f_{i} f\right) d \Omega+n(n-1) K^{2} \int_{\Omega} V f^{2} d \Omega .
\end{aligned}
$$

We deal with the terms $\int_{\Omega}-\bar{\Delta} f \sum_{i=1}^{n} V_{i} f_{i} d \Omega$ and $-(2 n-2) K \int_{\Omega} \sum_{i=1}^{n} V_{i} f_{i} f d \Omega$ in (9) by integration by parts again.

$$
\begin{aligned}
& \int_{\Omega}-\bar{\Delta} f \sum_{i=1}^{n} V_{i} f_{i} d \Omega=\int_{M}-f_{\nu} \sum_{i=1}^{n} V_{i} f_{i} d A+\int_{\Omega} \sum_{i, j=1}^{n} V_{i j} f_{i} f_{j}+\sum_{i=1}^{n} V_{i}\left(\frac{1}{2}|\bar{\nabla} f|^{2}\right)_{i} d \Omega \\
& =\int_{M}-f_{\nu} \sum_{i=1}^{n} V_{i} f_{i}+\frac{1}{2}|\bar{\nabla} f|^{2} V_{\nu} d A \\
& +\int_{\Omega} \sum_{i, j=1}^{n} V_{i j} f_{i} f_{j}-\frac{1}{2} \bar{\Delta} V|\bar{\nabla} f|^{2} d \Omega . \\
& \int_{\Omega} \sum_{i=1}^{n} V_{i} f_{i} f d \Omega=\int_{\Omega} \sum_{i=1}^{n} V_{i}\left(\frac{1}{2} f^{2}\right)_{i} d \Omega=\int_{M} \frac{1}{2} f^{2} V_{\nu} d A-\int_{\Omega} \frac{1}{2} f^{2} \bar{\Delta} V d \Omega .
\end{aligned}
$$

Inserting (10) and (11) into (9), we obtain

$$
\begin{aligned}
& \int_{\Omega} V\left((\bar{\Delta} f+K n f)^{2}-\left|\bar{\nabla}^{2} f+K f g\right|^{2}\right) d \Omega \\
& =\int_{M} V \bar{\Delta} f f_{\nu}+|\bar{\nabla} f|^{2} V_{\nu}-V \sum_{i=1}^{n} f_{i \nu} f_{i}+(2 n-2) K V f f_{\nu} \\
& \quad-f_{\nu} \sum_{i=1}^{n} V_{i} f_{i}-(n-1) K f^{2} V_{\nu} d A \\
& \quad+\int_{\Omega} \sum_{i, j=1}^{n} V_{i j} f_{i} f_{j}-\bar{\Delta} V|\bar{\nabla} f|^{2}-(2 n-2) K V|\bar{\nabla} f|^{2}+V \sum_{i, j=1}^{n} R_{i j} f_{i} f_{j} d \Omega \\
& +(n-1) \int_{\Omega}\left(K \bar{\Delta} V+K^{2} n V\right) f^{2} d \Omega .
\end{aligned}
$$

We now handle the boundary term in (12). We choose an orthonormal frame $\left\{e_{i}\right\}_{i=1}^{n}$ such that $e_{n}=\nu$ on $M$. Note that $z=\left.f\right|_{M}$ and $u=f_{\nu}$. From 
Gauss-Weingarten formula we deduce

$$
\begin{aligned}
& \int_{M} V \bar{\Delta} f f_{\nu}-V \sum_{i=1}^{n} f_{i \nu} f_{i} d A=\int_{M} V \sum_{i=1}^{n-1} f_{i i} f_{\nu}-V \sum_{i=1}^{n-1} f_{i \nu} f_{i} d A \\
= & \int_{M} V\left(u \Delta z+(n-1) H u^{2}-\langle\nabla u, \nabla z\rangle+h(\nabla z, \nabla z)\right) d A .
\end{aligned}
$$

On the other hand,

$$
\begin{aligned}
\int_{M}|\bar{\nabla} f|^{2} V_{\nu}-\sum_{i=1}^{n} f_{\nu} V_{i} f_{i} d A & =\int_{M}|\nabla z|^{2} V_{\nu}-u\langle\nabla V, \nabla z\rangle d A \\
& =\int_{M}|\nabla z|^{2} V_{\nu}+V\langle\nabla u, \nabla z\rangle+V u \Delta z d A(14)
\end{aligned}
$$

It follows from (13) and (14) that

$$
\begin{aligned}
& \int_{M} V \bar{\Delta} f f_{\nu}+|\bar{\nabla} f|^{2} V_{\nu}-V \sum_{i=1}^{n} f_{i \nu} f_{i}+(2 n-2) K V f f_{\nu} \\
& -f_{\nu} \sum_{i=1}^{n} V_{i} f_{i}-(n-1) K f^{2} V_{\nu} d A \\
= & \int_{M} V\left(2 u \Delta z+(n-1) H u^{2}+h(\nabla z, \nabla z)+(2 n-2) K u z\right) d A \\
& +\int_{M} V_{\nu}\left(|\nabla z|^{2}-(n-1) K z^{2}\right) d A .
\end{aligned}
$$

Combining (12) and (15), we arrive at (4).

\section{Alternative proof of Alexandrov's Theorem}

In this section, we prove Alexandrov's Theorem in $\mathbb{S}_{+}^{n}$ and $\mathbb{H}^{n}$ by using the general Reilly's formula (4).

For the case $\Omega \subset \mathbb{S}_{+}^{n}$, we choose $K=1$ and $V(x)=\cos r(x)$. For the case $\Omega \subset \mathbb{H}^{n}$, we choose $K=-1$ and $V(x)=\cosh r(x)$. In any case, we have $\bar{\nabla}^{2} V=-K V g$. The general Reilly's formula (4) reduces to

$$
\begin{aligned}
& \int_{\Omega} V\left((\bar{\Delta} f+K n f)^{2}-\left|\bar{\nabla}^{2} f+K f g\right|^{2}\right) d \Omega \\
= & \int_{M} V\left(2 u \Delta z+(n-1) H u^{2}+h(\nabla z, \nabla z)+(2 n-2) K u z\right) d A \\
& +\int_{M} \bar{\nabla}_{\nu} V\left(|\nabla z|^{2}-(n-1) K z^{2}\right) d A .
\end{aligned}
$$

Let $f: \Omega \rightarrow \mathbb{R}$ be the solution of

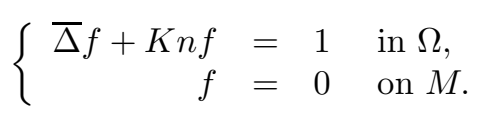

Then from (16) and Schwarz's inequality we obtain

$$
\frac{n-1}{n} \int_{\Omega} V d \Omega \geq \int_{M}(n-1) H u^{2} V d A .
$$


Since $H$ is a constant, we have from (17) and Hölder inequality that

$$
\int_{\Omega} V d \Omega \geq n H \int_{M} u^{2} V d A \geq n H \frac{\left(\int_{M} u V d A\right)^{2}}{\int_{M} V d A} .
$$

By Green's formula and $\bar{\Delta} V=-K n V$, we have

$$
\int_{M} u V d A=\int_{\Omega} \bar{\Delta} f V-f \bar{\Delta} V d \Omega=\int_{\Omega} V d \Omega .
$$

On the other hand, Minkowski formula in $\mathbb{S}_{+}^{n}$ or $\mathbb{H}^{n}$ tells that

$$
\int_{M} V d A=\int_{M} H p d A=H \int_{M} p d A=n H \int_{\Omega} V d \Omega .
$$

Here $p=\sin r\langle\bar{\nabla} r, \nu\rangle$ for $\Omega \subset \mathbb{S}_{+}^{n}$ and $p=\sinh r\langle\bar{\nabla} r, \nu\rangle$ for $\Omega \subset \mathbb{H}^{n}$.

Combining (17)-(20), we find equality holds in (17). Thus we must have $\left|\bar{\nabla}^{2} f+K f g\right|^{2}=\frac{1}{n}(\bar{\Delta} f+K n f)^{2}$. Taking into account that $\bar{\Delta} f+K n f=1$, we obtain

$$
\bar{\nabla}^{2}\left(f+\frac{1}{n}\right)=-K\left(f+\frac{1}{n}\right) g \text { in } \Omega .
$$

Since $f+\left.\frac{1}{n}\right|_{M}=\frac{1}{n}$, it follows from an Obata type result (see Reilly [11]) that $\Omega$ must be a geodesic ball. We complete the proof of Theroem 1.2 .

\section{Heintze-Karcher type inequality}

In this section, we prove Theorem 1.3 by using the general Reilly's formula (4).

We let $K=-1$ and $V(x)=\cosh r(x)$ in (4), $f$ be the solution to the following Dirichlet boundary value problem:

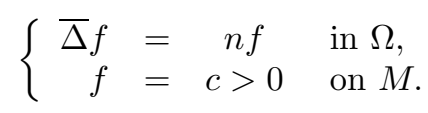

By assumption, Sect $\geq-1$, Hessian comparison theorem (see [13]) tells that $r(x)=\operatorname{dist}(x, p)$ satisfies

$$
\nabla^{2} r \leq \operatorname{coth} r\left(g-d r^{2}\right), \quad \text { for } x \in \Omega \backslash C u t(p),
$$

where $\operatorname{Cut}(p)$ is the cut locus of $p$. Thus

$$
\nabla^{2} V=\sinh r \nabla^{2} r+\cosh r d r^{2} \leq \cosh r g=V g, \quad \text { for } x \in \Omega \backslash C u t(p) .
$$

It follows that

$$
\Delta V g-\nabla^{2} V \leq(n-1) V g, \quad \Delta V \leq n V, \quad \text { for } x \in \Omega \backslash C u t(p) .
$$

Since $\operatorname{Cut}(p)$ has zero measure, we obtain from (23) and Ric $\geq-(n-1) g$ that

$$
\int_{\Omega}\left(\bar{\nabla}^{2} V-\bar{\Delta} V g+(2 n-2) V g+V R i c\right)(\bar{\nabla} f, \bar{\nabla} f) d \Omega \geq 0,
$$

and

$$
(n-1) \int_{\Omega}(-\bar{\Delta} V+n V) f^{2} d \Omega \geq 0
$$


Using (41), (24), (25), Schwarz's inequality and $\left.f\right|_{M}=c$, we have

$$
\begin{aligned}
0 & =\frac{n-1}{n} \int_{\Omega} V(\bar{\Delta} f-n f)^{2} d \Omega \\
& \geq \int_{\Omega} V\left((\bar{\Delta} f-n f)^{2}-\left|\bar{\nabla}^{2} f-f g\right|^{2}\right) d \Omega \\
& \geq \int_{M}(n-1) H u^{2} V-(2 n-2) c u V+(n-1) c^{2} \bar{\nabla}_{\nu} V d A .
\end{aligned}
$$

By Hölder inequality and (26) we deduce that

$$
\begin{aligned}
\left(\int_{M} u V d A\right)^{2} & \leq \int_{M} \frac{V}{H} d A \int_{M} H u^{2} V d A \\
& \leq \int_{M} \frac{V}{H} d A \int_{M} 2 c u V-c^{2} \bar{\nabla}_{\nu} V d A
\end{aligned}
$$

It follows that

$$
\begin{aligned}
& \left(\int_{M} u V d A-c \int_{M} \frac{V}{H} d A\right)^{2}-c^{2}\left(\int_{M} \frac{V}{H} d A\right)^{2} \\
& \leq-c^{2} \int_{M} \frac{V}{H} d A \int_{M} \bar{\nabla}_{\nu} V d A .
\end{aligned}
$$

Thus

$$
-c^{2}\left(\int_{M} \frac{V}{H} d A\right)^{2} \leq-c^{2} \int_{M} \frac{V}{H} d A \int_{M} \bar{\nabla}_{\nu} V d A
$$

Since $c \neq 0$, by eliminating $-c^{2} \int_{M} \frac{V}{H} d A$ from both sides of (26), we conclude

$$
\int_{M} \frac{V}{H} d A \geq \int_{M} \bar{\nabla}_{\nu} V d A=\int_{\Omega} \bar{\Delta} V d \Omega
$$

We now explore the equality case. If the equality holds in (30), then equality must hold in (26). It follows that $\left|\bar{\nabla}^{2} f-f g\right|^{2}=\frac{1}{n}(\bar{\Delta} f-n f)^{2}$. Taking into account of $\bar{\Delta} f=n f$, we obtain

$$
\bar{\nabla}^{2} f=f g \text { in } \Omega .
$$

Since $\left.f\right|_{M}=c$, it follows from an Obata type result (see Reilly [11]) that $\Omega$ must be a geodesic ball in a space form with constant sectional curvature -1 . We complete the proof of Theorem 1.3 .

For the case $\Omega \subset \mathbb{S}_{+}^{n}$, our method still applies to prove (3), that is

$$
\int_{M} \frac{\cos r}{H} d A \geq n \int_{\Omega} \cos r d \Omega
$$

Since the proof is almost the same, we only indicate the difference. First, we choose $K=1$ and $V(x)=\cos r(x)>0$ in (4) and $f$ be the solution to the Dirichlet boundary value problem:

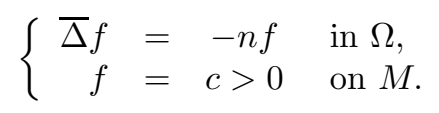


We notice that $\bar{\nabla}^{2} V=-V g$ and $\bar{\Delta} V=-n V$. Then the same argument as above works to show (28).

Acknowledgments. The research of the first author is supported by National Natural Foundation of China (Grant No. 11301497). The second author has received funding from the European Research Council under the European Union's Seventh Framework Programme (FP7/2007-2013) / ERC grant agreement no. 267087. Both authors would like to express their gratitude to Prof. Xinan Ma and Prof. Guofang Wang for their constant encouragement and support.

\section{References}

[1] A. D. Alexandrov: Uniqueness theorems for surfaces in the large I-V, Vestnik Leningrad Univ., 11, 5-17 (1956); 12, 15-44 (1957); 13, 14-26 (1958); 13, 27-34 (1958); 13, 5-8 (1958); English transl. in Amer. Math. Soc. Transl. 21 341-354, 354-388, 389-403, 403-411, 412-416 (1962).

[2] S. Brendle, Constant mean curvature surfaces in warped product manifolds, Publ. Math. Inst. Hautes Études Sci. 117, 247-269 (2013).

[3] S. Brendle, P.K. Hung and M.T. Wang, A Minkowski-type inequality for hypersurfaces in the Anti-deSitter-Schwarzschild manifold, arXiv:1209.0669.

[4] S. Brendle and M. T. Wang, A Gibbons-Penrose inequality for surfaces in Schwarzschild spacetime, arXiv:1303.1863.

[5] H. -I. Choi and A. -N. Wang, A first eigenvalue estimate for minimal hypersurfaces. J. Differential Geom. 18 no. 3, 559-562 (1983).

[6] L. de Lima and F. Girao, An Alexandrov-Fenchel-type inequality in hyperbolic space with an application to a Penrose inequality, arXiv:1209.0438.

[7] E. Heintze and H. Karcher, A general comparison theorem with applications to volume estimates for submanifolds, Ann. Sci. École Norm. Sup. 11, 451-470 (1978).

[8] S. Montiel and A. Ros, Compact hypersurfaces: The Alexandrov theorem for higher order mean curvatures, Pitman Monographs and Surveys in Pure and Applied Mathematics 52 (in honor of M.P. do Carmo; edited by B. Lawson and K. Tenenblat), 279-296 (1991).

[9] S. Pigola, M. Rigoli and A. G.Setti, Some applications of integral formulas in Riemannian geometry and PDE's, Milan J. Math. 71 219-281 (2003).

[10] R. C. Reilly, Applications of the Hessian operator in a Riemannian manifold, Indiana Univ. Math. J., 26, 459-472 (1977).

[11] R. C. Reilly, Geometric applications of the solvability of Neumann problems on a Riemannian manifold, Arch. Rational Mech. Anal. 75, no. 1, 23-29 (1980).

[12] A. Ros, Compact hypersurfaces with constant higher order mean curvatures, Revista Mathmática Iberoamericana, 3, 447-453 (1987).

[13] R. Schoen and S.-T. Yau, Lectures on differential geometry, International Press, Cambridge, MA, v+235 pp. (1994) 